\title{
Bringing ion channel crystal structures into sharper focus with computer modeling: examples from mechanosensitive channels
}

\begin{abstract}
"Over the past decade, increasing numbers of ion channel crystal structures have been solved, and many more structures will likely be determined in the coming years. Computational modeling can play an important role in verifying and helping to understand these channel crystal structures. This article focuses on a few important ways in which computer modeling has promoted the understanding of bacterial mechanosensitive ion channel crystal structures. Considering these studies of largeand small-conductance mechanosensitive ion channels provides general insights into the role computer modeling can play in elucidating aspects of channel structures that are essential in drug-design efforts."
\end{abstract}

Increasing numbers of ion channel crystal structures have been solved over the past decade, providing significant insights into channel function on the molecular level. In particular, the availability of channel structures can greatly promote the rational design of drug molecules targeted to channels. Despite their importance, crystal structures only provide a static image of a channel under conditions that are typically rather unphysiological. For example, most membrane proteins are crystallized in the absence of membrane lipids. In addition, the structures of many membrane proteins are solved at a relatively low resolution. These issues can lead to concerns about the conformation or physiological relevance of a channel structure, thus obscuring its interpretation. Certainly, researchers can use a variety of experimental methods, such as electron paramagnetic resonance (EPR) [1], chemical crosslinking [2] and fluorescence resonance energy transfer (FRET) [3] to address these ambiguities. However, computer modeling also plays a central role in bringing the interpretation of channel structures into clear focus, elucidating their relevance to channel function.

This article describes the central role played by computer modeling in studies of two bacterial ion channels, the mechanosensitive channels of large (MscL) and small (MscS) conductance. In bacteria, these channels are thought to provide protection from osmotic downshock [4]. To date, these are the bestcharacterized mechanosensitive channels [5] and the existence of high-resolution structures of both channels [6-10] gave them the potential to serve as important models for mechanosensation on the molecular level. Mechanosensitive channels are important in a wide variety of physiological processes in higher organisms, including hearing, touch and circulation [4]. Any attempt to design therapeutic agents targeting these mechanosensitive channels would benefit from a detailed molecular-level understanding of mechanosensitivity.

\section{“Despite their importance, crystal structures only provide a static image of a channel under conditions that are typically rather unphysiological."}

Despite the potential utility of crystal structures in understanding MscL and MscS, there were significant questions about the conformations of these structures and their physiological relevance. The three following vignettes describe how computational methods were critical in addressing these ambiguities. By helping researchers verify and interpret these structures, computational methods played an essential role in helping MscL and MscS structures to serve as effective model systems for mechanosensitivity. The studies described are not intended to be a comprehensive review of ion channel computations or studies of mechanosensitive channels, which have both been the subject of recent reviews [11-13]. Instead, they were chosen as brief case studies to provide insights into how computational methods can help researchers

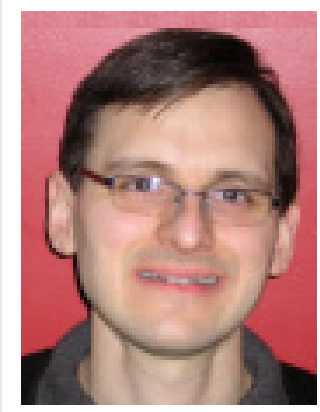

Donald E Elmore

Wellesley College, Department of Chemistry, 106 Central Street,

Wellesley, MA 0248I, USA

Tel.: + $781283317 \mid$

Fax: +| 78| 2833642

E-mail: delmore@wellesley.edu 
understand and practically use crystal structure information obtained for channels that are relevant in pharmaceutical design.

\section{The C-terminal region of MscL}

Computer modeling has played a key role in identifying issues in the initial crystal structure of $\mathrm{MscL}$, proposing alternative structures and verifying a revised version of the MscL crystal structure. When the structure of the MscL homologue from Mycobacterium tuberculosis was first solved by the Rees group in 1998 [7], it showed a distinct intracellular C-terminal domain consisting of an $\alpha$-helical bundle. While this domain was initially thought to be unimportant for channel function [14], more recent studies have noted that mutations to charged residues and cleavages in the region do affect channel physiology [15,16]. Thus, the C-terminal region has a clear physiological role, even if it is not fully understood.

Despite the interest in this C-terminal region, its structure in the initial MscL crystal structure was suspect. In particular, the crystal structure conformation buried several negatively charged Asp and Glu residues in close proximity, while exposing hydrophobic amino acids to water. Molecular dynamic (MD) simulations were used to consider the stability of this conformation [17]. MD simulations use molecular mechanics force fields to model how a system moves over a period of time. Using an MD simulation, one can consider the structural stability of a protein. Simulations clearly showed that the $\mathrm{C}$-terminal $\alpha$-helical bundle in the MscL crystal structure was not structurally stable, as it rapidly lost its structure during a simulation [17]. Interestingly, circular dichroism spectra of a model system containing the C-terminal sequence of MscL implied that the region nonetheless had an $\alpha$-helical conformation [18]. This led Anishkin et al. to propose an alternative $\alpha$-helical bundle conformation for the C-terminal region of the Escherichia coli homolog of MscL, based on its homology with the cartilage oligomeric matrix protein (COMP) [15]. This alternative conformation was stable in MD simulations and was further supported by experimental cysteine crosslinking data [15].

After these modeling studies were completed, Rees and co-workers released a revised version of the $M$. tuberculosis MscL crystal structure that included a C-terminal conformation quite similar to that proposed for E. coli MscL [8]. MD simulations were again used to verify this revised structure [19]. These simulations showed that the new crystal structure conformation was quite stable under the same simulation conditions that had led to significant degradation of the initial crystal structure conformation, supporting the validity of the revised structure. The revised conformation was also consistent with experimental data on the stability of mutants in the C-terminal region [19].

\section{Determining the physiological state of the MscS crystal structure}

Most crystal structures capture ion channels in their closed conformation. While this is not surprising since open states typically represent conformations that would not be promoted under crystallization conditions, it is possible to obtain the structure of a channel in its open state $[10,20]$. These structures of conductive states are clearly desirable for understanding channel-gating mechanisms. Thus, it was particularly notable when the crystal structure of $\mathrm{MscS}$ published by Bass et al. had a wide pore region that was only approximately $10 \AA$ in diameter at its narrowest point [6]. Since this opening appeared large enough to pass ions, researchers initially assumed that the structure represented the open state of the channel.

Computational modeling allowed a variety of groups to investigate more rigorously whether the pore in the MscS crystal structure could actually conduct ions. In an initial MD study, Sukharev and co-workers performed simulations of the transmembrane region of the channel with its backbone restrained to the crystal structure conformation [21]. Interestingly, they observed that the channel was unable to maintain aqueous hydration in this conformation, as the channel was lined with several hydrophobic residues. Not surprisingly, this lack of hydration throughout the pore provided a significant barrier to ion permeation. While intriguing, these simulations only considered the transmembrane regions of the channel with membrane modeled as octane solvent, and these simplifications could have led to a spurious result. However, similar results were observed in subsequent simulations that utilized the whole channel structure embedded in a fully represented lipid membrane [22-24]. Simulations in all these studies showed very low conductance of the channel when it was restrained to its crystal structure conformation, implying that the structure may not represent an open state. 
One limitation of all-atom MD simulations, such as those described in the last paragraph for $\mathrm{MscS}$, is that they can only model a relatively short timescale, typically in the 10-100-ns range for large channel systems. In fact, all the individual simulations of $\mathrm{MscS}$ included in the studies described previously were less than $10 \mathrm{~ns}$ in length. Other computational methods that utilize a simplified model of the system can provide information over a longer timescale. In the case of MscS, other studies used Brownian Dynamics (BD) [25] and Biology Monte Carlo (BioMOCA) [26] methods to consider the flow of ions through the channel. In both of these methods, the protein, membrane and aqueous solvent are modeled as continuum regions that do not move during the simulation. Thus, only the movement of ions is calculated over the course of the simulation, greatly increasing the speed of the calculation. For MscS, multiple simulations of $100 \mathrm{~ns}$ or greater were performed with BioMOCA [26] and simulations longer than $1 \mu$ s were published using BD [25]. Both of these methods largely agreed with the MD results, showing that the MscS crystal structure conformation yielded a dramatically lower conductivity than observed experimentally for the channel. The agreement between these quite different computational methods was particularly satisfying and showed the robustness of the assertion that the MscS crystal structure did not represent the open state of the channel.

If the crystal structure was not of the open state of MscS, then what state did it represent? MD simulations implied that this structure was not the fully closed state since an unrestrained channel in simulations rapidly collapsed into a state with a fully occluded pore in the absence of applied tension or potential [22,23]. Thus, it seems that the crystal structure conformation was not a stable closed state of the channel. However, MD studies also showed that the channel could assume a conductive conformation with relatively small structural adjustments in the presence of membrane tension [22] or a transmembrane potential [23,24], although the conductance and ion selectivity under these conditions did not necessarily match experimental measurements. Thus, it seemed that the crystal structure conformation was at least close in conformation to a conductive state, and could potentially represent an intermediate or desensitized state of $\mathrm{MscS}$, although the exact physiological state of this structure remains unknown.

\section{Modeling the closed \& open states of MscS}

Even if a crystal structure represents a potentially nonphysiological state of an ion channel, as may have been true for the initial MscS structure, it can nonetheless serve as a useful starting point for developing models of physiologically relevant open and closed states. In fact, computer modeling methods were applied to the MscS crystal structure to propose closed and open state models of that channel. While the MD simulations described earlier led to a collapsed form of MscS [22,23], this collapsed form would not necessarily represent the true closed state of the channel. For example, these conformations did not necessarily agree with experimental crosslinking studies performed on the closed state of the channel [27].

\section{"Even if a crystal structure represents a potentially nonphysiological state of an ion channel ... it can nonetheless serve as a useful starting point for developing models of physiologically relevant open and closed states."}

Starting with the $\mathrm{MscS}$ crystal structure, two different groups developed closed-state models of MscS. Sukharev and co-workers developed a more compacted closed state of the channel using an iterative approach with energy minimizations and short MD simulations [28]. The closed-state model that resulted from this approach was significantly more stable than the crystal structure when subjected to MD simulations in explicit membrane without any applied stimulus, supporting the assertion that it represented the stable closed state of the channel. Moreover, this structure was consistent with experimental cysteine crosslinking results performed in conjunction with the modeling [28]. Perozo and co-workers derived a closed-state model by combining computer modeling with experimental data [29]. In this approach, they obtained data on the water and lipid accessibility for each residue in the transmembrane region of $\mathrm{MscS}$ using EPR spin labeling. They were then able to use these data to place restraints on MD simulations that were used to produce their closed-state model. This closed-state model was also stable in an unrestrained MD simulation in a lipid membrane [29]. These research groups also used similar approaches to develop models of $\mathrm{MscS}$ in the open state [30,31]. Despite being derived from quite different methods, these 
open-state models share significant similarities, such as in the pore-lining transmembrane domain (TM3). After these models were developed, a crystal structure of a single-site mutant of $\mathrm{MscS}$ was solved that appears to be in the open state [10]. Although some features differ between this structure and the models, such as the exact tilt of the pore-lining domains, the open-state models and the structure share many notable features.

\section{“...verifying that a crystal structure conformation is actually stable under physiological conditions would be desirable before using the structure as the basis for extensive drug design."}

Clearly, the existence of MscS models and structures in different physiological states will allow further experimental and computational studies to delve into the gating pathway in more detail. For example, coarse-grain simulations, which have been used recently to consider MscL and $\mathrm{MscS}[32,33]$, can allow one to consider protein dynamics, such as the progression between different conformations, over a longer timescale than traditional all-atom MD simulations.

\section{Conclusion}

While atomic-resolution crystal structures can appear to provide all the answers about how proteins work, there are still significant questions about how these structures relate to the physiological function of proteins. The three sets of studies described in this article demonstrate how computational modeling played a significant role in helping researchers verify and understand crystal structures of mechanosensitive channels.
Computations will play a similar role in clarifying questions about the numerous crystal structures of pharmaceutically relevant ion channels that are certain to be solved in the coming years, bringing these structures into sharper focus for medicinal chemists who will use them in drugdesign efforts. For example, verifying that a crystal structure conformation is actually stable under physiological conditions would be desirable before using the structure as the basis for extensive drug design. Furthermore, knowing whether a structure corresponds to the open or closed state of a channel would be critical to understanding how a drug targeted to that structure would affect channel function. As innovations in computer processor speed make increasingly complex simulations feasible and experimental efforts determine increasing numbers of membrane protein structures, this important interplay between crystal structures and computer modeling will be enhanced in the coming years.

\section{Acknowledgements}

The author would like to thank Joshua A Maurer, Steve A Spronk, Li Xiong, Dennis A Dougherty and Henry A Lester for interesting discussions throughout our collaborations on mechanosensitive channels.

\section{Financial \& competing interests disclosure}

Research on these systems performed by the author was funded by a NIH program project grant (GM 62532) and the Office of the Dean of Wellesley College, MA, USA. The author has no other relevant affliations or financial involvement with any organization or entity with a financial interest in or financial conflict with the subject matter or materials discussed in the manuscript apart from those disclosed.

No writing assistance was utilized in the production of this manuscript.

\section{Bibliography}

1 Perozo E, Cuello LG, Cortes DM, Liu YS, Sompornpisut P. EPR approaches to ion channel structure and function. Novartis Found. Symp. 245, 146-158 (2002).

2 Bass RB, Butler SL, Chervitz SA, Gloor SL, Falke JJ. Use of site-directed cysteine and disulfide chemistry to probe protein structure and dynamics: applications to soluble and transmembrane receptors of bacterial chemotaxis. Methods Enzymol. 42, 325-351 (2007).

3 Machiyama H, Tatsumi H, Sokabe M. Structural changes in the cytoplasmic domain of the mechanosensitive channel MscS during opening. Biophys. J. 97(4), 1048-1057 (2009).
4 Hamill OP, Martinac B. Molecular basis of mechanotransduction in living cells. Physiol. Rev. 81(2), 685-740 (2001).

5 Corry B, Martinac B. Bacterial mechanosensitive channels: experiment and theory. Biochim. Biophys. Acta 1778(9), 1859-1870 (2008).

6 Bass RB, Strop P, Barclay M, Rees DC. Crystal structure of Escherichia coli $\mathrm{MscS}$, a voltage-modulated and mechanosensitive channel. Science 298(5598), 1582-1587 (2002).

7 Chang G, Spencer RH, Lee AT, Barclay MT, Rees DC. Structure of the MscL homolog from Mycobacterium tuberculosis: a gated mechanosensitive ion channel. Science 282(5397), 2220-2226 (1998).
8 Steinbacher S, Bass R, Strop P, Rees DC. Structures of the prokaryotic mechanosensitive channels MscL and MscS. Curr. Topics Membr. 581-524 (2007).

9 Liu Z, Gandhi CS, Rees DC. Structure of a tetrameric MscL in an expanded intermediate state. Nature 461(7260), 120-124 (2009).

10 Wang W, Black SS, Edwards MD et al. The structure of an open form of an E. coli mechanosensitive channel at $3.45 \AA$ resolution. Science 321(5893), 1179-1183 (2008).

11 Corry B, Martinac B. Bacterial mechanosensitive channels: experiment and theory. Biochim Biophys Acta 1778(9), 1859-1870 (2008). 
12 Khalili-Araghi F, Gumbart J, Wen PC, Sotomayor M, Tajkhorshid E, Schulten K. Molecular dynamics simulations of membrane channels and transporters. Curr. Opin. Struct. Biol. 19(2), 128-137 (2009).

13 Lindahl E, Sansom MS. Membrane proteins: molecular dynamics simulations. Curr. Opin. Struct. Biol. 18(4), 425-431 (2008).

14 Blount P, Sukharev SI, Schroeder MJ, Nagle SK, Kung C. Single residue substitutions that change the gating properties of a mechanosensitive channel in Escherichia coli. Proc. Natl Acad. Sci. USA 93(21), 11652-11657 (1996).

15 Anishkin A, Gendel V, Sharifi NA et al. On the conformation of the $\mathrm{COOH}$-terminal domain of the large mechanosensitive channel MscL. J. Gen. Physiol. 121(3), 227-244 (2003).

16 Kloda A, Ghazi A, Martinac B. C-terminal charged cluster of MscL, RKKEE, functions as a pH sensor. Biophys. J. 90(6), 1992-1998 (2006).

17 Elmore DE, Dougherty DA. Molecular dynamics simulations of wild-type and mutant forms of the Mycobacterium tuberculosis MscL channel. Biophys. J. 81(3), 1345-1359 (2001).

18 Kochendoerfer GG, Tack JM, Cressman S. Total chemical synthesis of a $27 \mathrm{kDa}$ TASP protein derived from the MscL ion channel of M. tuberculosis by ketoxime-forming ligation. Bioconjugate Chem. 13(3), 474-480 (2002).

19 Maurer JA, Elmore DE, Clayton D, Xiong L, Lester HA, Dougherty DA. Confirming the revised C-terminal domain of the MscL crystal structure. Biophys. J. 94(12), 4662-4667 (2008).
20 Long SB, Campbell EB, Mackinnon R. Crystal structure of a mammalian voltagedependent Shaker family $\mathrm{K}^{+}$channel. Science 309(5736), 897-903 (2005).

21 Anishkin A, Sukharev S. Water dynamics and dewetting transitions in the small mechanosensitive channel MscS. Biophys. J. 86(5), 2883-2895 (2004).

22 Sotomayor M, Schulten K. Molecular dynamics study of gating in the mechanosensitive channel of small conductance MscS. Biophys. J. 87(5), 3050-3065 (2004).

23 Spronk SA, Elmore DE, Dougherty DA. Voltage-dependent hydration and conduction properties of the hydrophobic pore of the mechanosensitive channel of small conductance. Biophys. J. 90 (10), 3555-3569 (2006).

24 Sotomayor M, Vasquez V, Perozo E, Schulten K. Ion conduction through MscS as determined by electrophysiology and simulation. Biophys. J. 92(3), 886-902 (2007).

25 Vora T, Corry B, Chung SH. Brownian dynamics investigation into the conductance state of the MscS channel crystal structure. Biochim. Biophys. Acta 1758(6), 730-737 (2006).

26 Sotomayor M, van der Straaten TA, Ravaioli U, Schulten K. Electrostatic properties of the mechanosensitive channel of small conductance MscS. Biophys. J. 90(10), 3496-3510 (2006).
27 Miller S, Edwards MD, Ozdemir C, Booth IR. The closed structure of the MscS mechanosensitive channel. Cross-linking of single cysteine mutants. J. Biol. Chem. 278(34), 32246-32250 (2003).

28 Anishkin A, Akitake B, Sukharev S. Characterization of the resting MscS: modeling and analysis of the closed bacterial mechanosensitive channel of small conductance. Biophys. J. 94(4), 1252-1266 (2008).

29 Vasquez V, Sotomayor M, Cortes DM, Roux B, Schulten K, Perozo E. Three-dimensional architecture of membrane-embedded $\mathrm{MscS}$ in the closed conformation. J. Mol. Biol. 378(1), 55-70 (2008).

30 Anishkin A, Kamaraju K, Sukharev S. Mechanosensitive channel MscS in the open state: modeling of the transition, explicit simulations, and experimental measurements of conductance. J. Gen. Physiol. 132(1), 67-83 (2008).

31 Vasquez V, Sotomayor M, Cordero-Morales J, Schulten K, Perozo E. A structural mechanism for $\mathrm{MscS}$ gating in lipid bilayers. Science 321(5893), 1210-1214 (2008).

32 Yefimov S, van der Giessen E, Onck PR, Marrink SJ. Mechanosensitive membrane channels in action. Biophys. J. 94(8), 2994-3002 (2008).

33 Li S, Zhang X, Wang W. Coarse-grained model for mechanosensitive ion channels. J. Phys. Chem. B 113(43), 14431-14438 (2009). 